\section{Systemic lupus erythematosus flare up as acute spinal subarachnoid hemorrhage with bilateral lower limb paralysis}

\author{
Xiang Yang, ${ }^{1}$ Seidu A. Richard, ${ }^{1-3}$ \\ Jiagang Liu, ${ }^{1}$ Siqing Huang ${ }^{1}$ \\ ${ }^{1}$ Department of Neurosurgery, West \\ China Hospital, Sichuan University, P.R \\ China; ${ }^{2}$ Department of Immunology, \\ Jiangsu University, Jiangsu, P.R. China; \\ ${ }^{3}$ Department of Surgery, Volta Regional \\ Hospital, Ho, Ghana, West Africa
}

\begin{abstract}
Subarachnoid hemorrhage (SAH) is an uncommon complication of systemic lupus erythematosus (SLE). Solitary association of fatal spinal SAH as a complication of SLE, has not been encountered much in literature although coexisting acute cerebral and spinal SAH have been associated with SLE. We present a 39-year old female with initial diagnosis of SLE eight years ago who suddenly developed a productive cough, acute abdomen and paralysis of the lower limbs. Magnetic resonance imaging of the spine revealed thoracic spinal SAH with varying degrees of thoracic spinal cord compression. The hemorrhage was total evacuated via surgery. She regained normal function of her lower limbers after the operation with no further neurological complications. One of the rare but fatal complications of SLE is solitary spinal SAH without cranial involvement. The best and most appropriate management of this kind of presentation is surgical decompression of the hematoma with total hemostasis. The cause of hemorrhage should be identified intra-operatively and treated appropriately.
\end{abstract}

\section{Introduction}

Systemic lupus erythematosus (SLE) is an autoimmune disease associated with complex-mediated lesions of blood vessels in multiple body organs, advancing to diverse kinds of vascular complications. ${ }^{1-3}$ Immune complexes that trigger vasculitis in SLE may contain DNA and Anti-DNA antigens, or Ro and Anti-Ro antigens and frequently involves small vessels with occasional involvement of medium and large vessels..$^{1,4}$ Although cranial Subarachnoid hemorrhage (SAH) is generally recognized as a major complication of $\mathrm{SLE}^{2,5,6}$ and coexisting acute cerebral and spinal SAH rarely reported in $\mathrm{SLE},{ }^{2}$ only one fatal spinal SAH as a complication of SLE, has not been encountered in literature. ${ }^{3} \mathrm{We}$ therefore present a case of SLE that flared up into acute spinal SAH with bilateral limb paralysis without cranial involvement.

\section{Ethics approval and consent to par- ticipate}

The ethical committee of the hospital full approved our case study. The patient and her relatives were informed about our intension to involve her in a case study and she/they agreed to partake in the study. She/they signed the concern form before the operation was carried out according to all surgical protocols.

\section{Consent for publication}

The patient and his relatives were dually informed about our intention to publish her case and she/they fully concerted to the use of his documents. The hospital also concerted to the use of her information for publication.

\section{Case Report}

We present a 39-year old female with progressive multiple joint swelling associated with pain for eight years. Her symptoms become much severe two years ago and suddenly progressed into a productive cough and acute abdomen with paralysis of the lower limbs days (2) before she arrived at our outfit. She has a past history of suspicious cat scratch disease. Physical examination revealed thinning of hair, dry skin and mucosa membranes with ecchymosis and petechial rashes. Superficial lymph nodes were palpable but not significantly enlarged. There were rashes all over the face with no oral ulcers. Chest examination revealed shallow movements with bronchial breath sounds associated with wet rales. There was marked edema of lower extremities with multiple joint tenderness on palpation. There was sensory loss up to the xiphoid with saddle areas of numbness. The power on left leg was $3 / 5$ while that of the right leg was $0 / 5$. Babinski examination revealed up moving planter reflex.

Magnetic resonance imaging (MRI) revealed thoracic spinal SAH with varying degrees of thoracic spinal cord compression (Figure 1A and B). The nerve root of T5-T7 were compressed by the hemorrhage. CTscan of the spine also confirmed hematoma (Figure 2A). Chest X-ray show congested lungs with patch opacities. Sputum examination did not yield any pathological organisms as the cause of the lung infection.
Correspondence: Jiagang Liu, Department of Neurosurgery, Post-Graduate Training Centre, West China Hospital, Sichuan University, 37 Guo Xue Xiang Road, Chengdu, Sichuan, 610041, P.R. China.

Tel.: +86.18980601975 - Fax: +86.02885422490 .

E-mail: neurovip@yeah.net

Key words: Subarachnoid hemorrhage; systemic lupus erythematosus; flare up; spinal; paralysis.

Contributions: SAR and XY conceived the project and SAR designed the study. SAR and XY collected patient's data. JL and SH provided technical assistance in the study. SAR analyzed the data, prepared the illustrations and wrote the paper. All authors approved the paper for the submission.

Conflict of interest: the authors declare no conflict of interest.

Received for publication: 8 February 2017.

Revision received: 6 March 2018.

Accepted for publication: 3 April 2018.

This work is licensed under a Creative Commons Attribution NonCommercial 4.0 License (CC BY-NC 4.0).

CC Copyright X. Yang et al., 2018

Licensee PAGEPress, Italy

Clinics and Practice 2018; 8:1069

doi:10.4081/cp.2018.1069

Liver function test and renal function test were normal. The hemoglobin was $77 \mathrm{~g} / \mathrm{L}$ indication anemia. Hepatitis B and C, HIV as well as syphilis serolog ies was all negative. Full immunization investigation revealed positive antinuclear antibodies (ANA + ), immunoglobulin $\mathrm{G}(\mathrm{IgG}): 17.60$ $\mathrm{g} / \mathrm{L}$, immunoglobulin A $(\operatorname{IgA}): 4.09 \mathrm{~g} / \mathrm{L}$, complement C3: $0.69 \mathrm{~g} / \mathrm{L}$. T-Score: -0.3 . Anti-human globulin test: direct Coombs test: positive $(++++)$, and indirect Coombs test: positive $(++)$. Rheumatoid factor $(\mathrm{RF})$ $<20.0 \mathrm{I} \mathrm{U} / \mathrm{mL}, \mathrm{C}$-reactive protein (CRP) $3.96 \mathrm{mg} / \mathrm{L}$, anti-streptolysin O (ASO) 28.90I U/mL, anti-cyclic citrullinated peptide antibody (CCP) $45.7 \mathrm{U} / \mathrm{mL}$ while antikeratin antibodies (AKA) was negative (-). Erythrocyte sedimentation rate (ESR) was $60 \mathrm{~mm} / \mathrm{h}$. Our working diagnosis based on radiological findings and laboratory investigation above was spina SAH secondary to systemic lupus erythematosus.

The goal of surgery in this patient was to evacuate the blood that was causing the compressive symptoms while preserving the nerve root and also prevent recurrence of the hemorrhage. The patient was put on a prone position after general anesthesia. After marking the location of the hemor- 
rhage and draping with povidone iodine, a $10-\mathrm{cm}$ midline incision was made between T5 and T7 and extended to the subcutaneous, intramuscular up to the vertebrates. Osteotomy of the T5-T7 was done with high-speed drill through the lamina of the vertebrates. Further bone and ligament removal was done using the Kerrison rongeur. After securing hemostasis, the blood was seen superficially around the spinal nerve root and spinal cord to the right. The blood was complete evacuated without causing damage to the spinal nerve root during the surgery (Figure 2B). The muscles, subcutaneous tissues and the skin was closed in layers and wound dressing applied. Intra-operatively we notice the blood was extra-dura but compressing on the nerve roots. The patient had a good recovering. Post-operative CT scan done the next day shows no bleeding. The limb function gradually improved. Patient was discharge home two weeks after the operation by which time she could walk with support. A scheduled visit three months later revealed total recovery.

\section{Discussion}

Neurologic problems are common in cases of SLE, affecting up to $75 \%$ of patients. ${ }^{3}$ The symptoms include seizures, cranial nerve disorders, paralysis, peripheral neuropathy, and mental changes. These symptoms may wax and wane during the course of the disease. ${ }^{3}$ SAH either cranial or spinal is a potentially lethal complication in patients with SLE. ${ }^{2,7,8}$ Our case adds up to the few unusual presentations of SLE flaring up as acute spinal SAH with bilateral limb paralysis without cranial involvement. The first case was reported by Fody et al. in $1980 .^{3}$ Cerebral vascular pathological changes as a result of autoimmunity in SLE patients includes cerebral thrombosis, cerebral infarction and cerebral hemorrhage which are serious threat to patients with SLE. ${ }^{9-12}$ We believe the cause of this spontaneous hemorrhage in our patient is due to thrombosis of bridging vessels as result of vasculitis.

Hemorrhage affecting the spinal cord in the general population is rare, and delayed diagnosis is often associated with poor outcome. $^{3}$ Common causes of spinal SAH reported in literature includes arteriovenous fistula, syphilis, polyarteritis nodosa, and aortic coarctation. ${ }^{3}$ Other causes of spontaneous subarachnoid hemorrhage of the cord include angioma and telangiectasis, solitary aneurysm, coarctation of the aorta, mycotic aneurysm of a spinal artery, various tumors (ependymoma, schwannoma, neurofibro- ma, glioblastoma multiforme, meningeal sarcoma) as well as anticoagulant therapy. ${ }^{3}$ In addition, various infections and blood dyscrasias are said to produce spinal subarachnoid hemorrhage although specific cases have not been documented. ${ }^{3}$ Spinal angioma is the most common cause of spontaneous spinal subarachnoid hemorrhage, occurring in $13 \%$ of patients. ${ }^{3} \mathrm{~A}$ review of the literature found only one report of spinal SAH in a 50-year-old woman with an 18-year history of $\mathrm{SLE}^{2,3}$ who died after an event of SLE flare up, and autopsy revealed massive spinal SAH due to vasculitis of the posterior spinal artery. ${ }^{2}$ Our case therefore becomes the second soli-

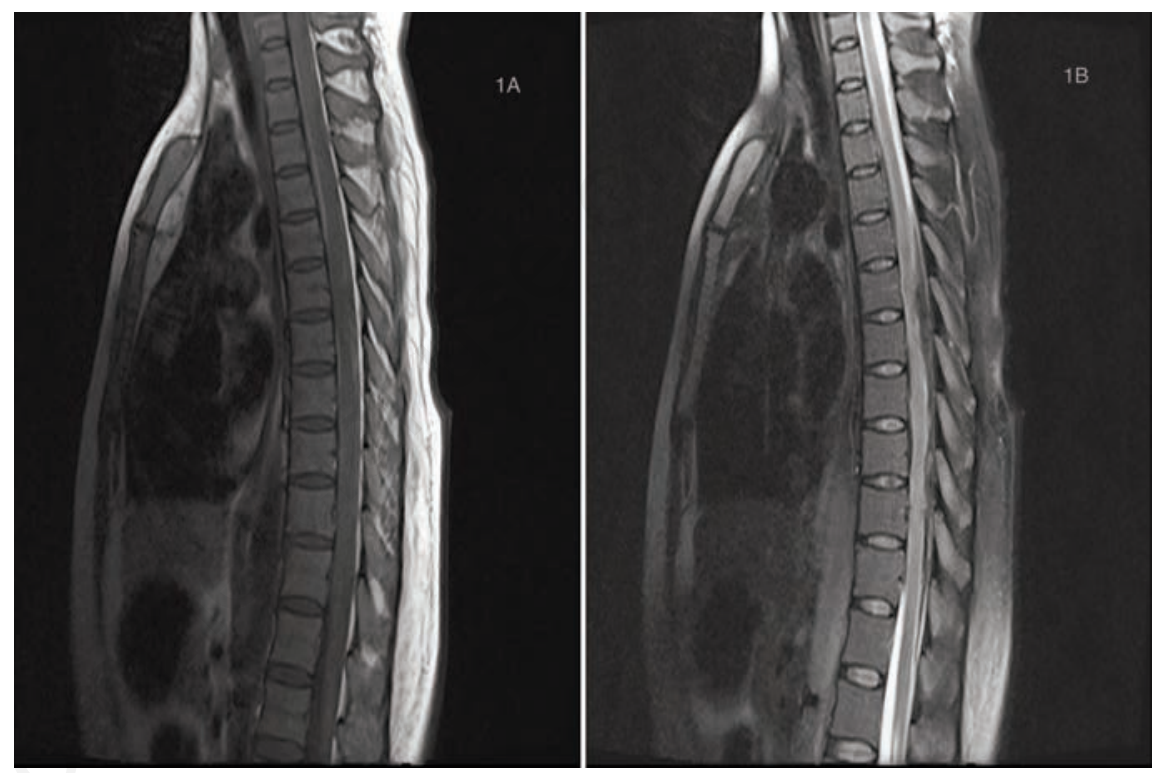

Figure 1. A) T1 and B) T2 magnetic resonance imaging respectively showing subarachnoid hemorrhage with spinal cord and never root compression.

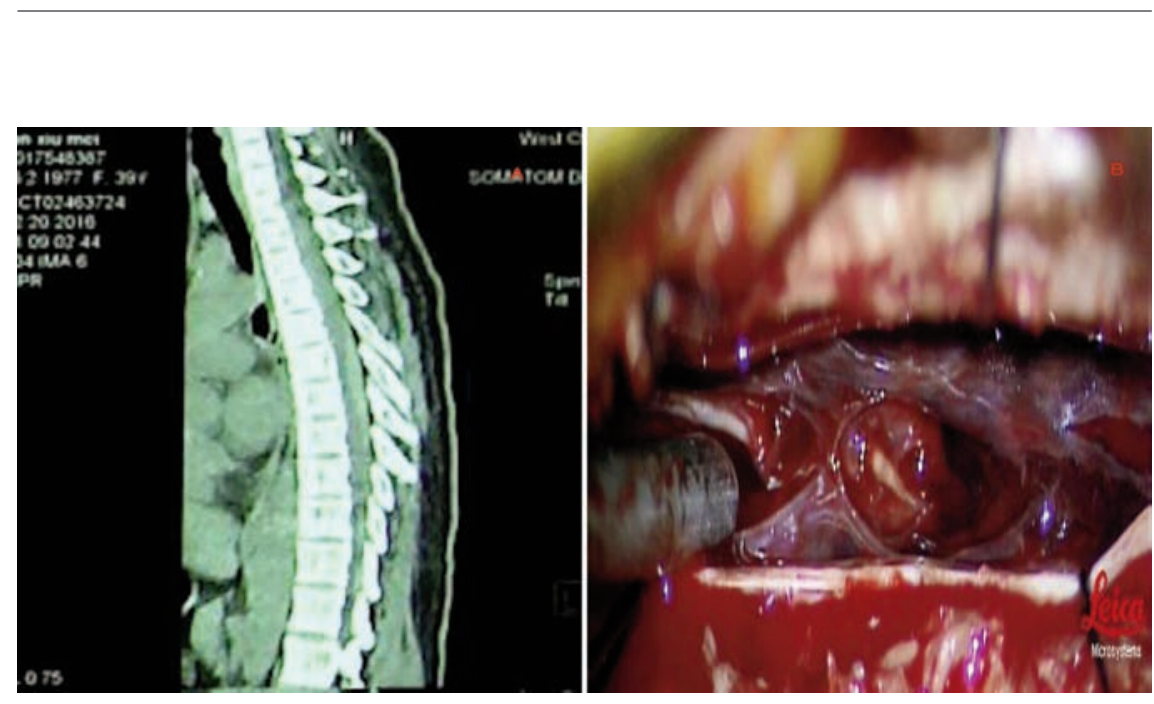

Figure 2. A) Computed tomographic scan image confirming subarachnoid hemorrhage; B) intraoperative image showing total evacuation of the hemorrhage. tary spinal subarachnoid hemorrhage as a complication of SLE. Acute severe back pain and headaches followed by symptoms of transverse myelopathy is usually the clinical manifestation of Spinal subarachnoid hemorrhage. ${ }^{3}$ It has been suggested that decreased CSF glucose level is characteristically associated with SLE induced transverse myelopathy, but spinal SAH also may produce this finding. ${ }^{3}$

Tang et al with a case SLE flaring up into cerebral and spinal SAH revealed that to the lumbar canal with maxima spine on the MRI. ${ }^{2}$ They further indicated the spinal SAH was from the posterior fossa 
that digital subtraction angiography (DSA) showed diffuse irregular narrowing and some occlusion of the medium and small arteries of the intracranial and spinal vasculature, suggesting vasculitis. ${ }^{2}$ Edward et al. with a similar case noted at autopsy that the spinal cord contained a large adherent blood clot dorsally, covering much of the thoracic region and extending to the cervical and lumbar levels, but not including the site of lumbar puncture. Lesions were not noted macroscopically. The hemorrhage ended abruptly at the foramen magnum. ${ }^{3}$

\section{Conclusions}

One of the rare but fatal complications of SLE is solitary spinal SAH without cranial involvement. The best treatment option in our case after careful evaluation was spinal decompressing of the acute hemorrhage; identification the cause of the hemorrhagic and tacking it appropriately was the very crucial part of the surgery. We managed the patients so as to avoid recurrence of the subarachnoid hemorrhage. After surgery, we recommend that patient be referred to rheumatologist to continue the management.

\section{References}

1. Torné R, Rodríguez-Hernández A, Bernard T, et al. Subarachnoid hemorrhage in systemic lupus erythematosus: Systematic review and report of three cases. Clin Neurol Neurosur 2015;128:17-24.

2. Tang S, Lee C, Lee C, Jeng J. Systemic lupus erythematosus flare up manifestation as cerebral and spinal subarachnoid hemorrhage. Lupus 2011;20:1211-3.

3. Fody EP, Netsky MG, Mrak RE. Subarachnoid spinal hemorrhage in a case of systemic lupus erythematosus. Archiv Neurol 1980;37:173-4.

4. Ramos-Casals M, Nardi N, Lagrutta M, et al. Vasculitis in systemic lupus erythematosus: prevalence and clinical characteristics in 670 patients. Medicine 2006;85:95-104.

5. Futrell N, Millikan C. Frequency, etiology, and prevention of stroke in patients with systemic lupus erythematosus.
Stroke 1989;20:583-91.

6. Bernatsky S, Clarke A, Gladman D, et al. Mortality related to cerebrovascular disease in systemic lupus erythematosus. Lupus 2006;15:835-9.

7. Owada T, Takahashi K, Kita Y. Subarachnoid hemorrhage in systemic lupus erythematosus in Japan: two case reports and a review of the literature. Modern Rheumatol 2009;19:573.

8. Baizabal Carvallo J, Cantú Brito C, Estañol B, García Ramos G. Subarachnoid hemorrhage as a complication of systemic lupus erythematosus. Cerebrovasc Dis 2007;24:301-4.

9. Zhong W, Chen H, You C, et al. Spontaneous spinal epidural hematoma. J Clin Neurosci. 2011;18:1490-4.

10. Penar P, Fischer D, Goodrich I, et al. Spontaneous spinal epidural hematoma. Int Surg 1986;72:218-21.

11. Baek BS, Hur JW, Kwon KY, Lee HK. Spontaneous spinal epidural hematoma. J Korean Neurosurg Soc 2008;44:40-2.

12. Matsumae M, Shimoda M, Shibuya N, et al. Spontaneous cervical epidural hematoma. Surg Neurol 1987;28:381-4. 